\title{
Stimulus encoding selectivity at high degrees of paired-associate learning*
}

\author{
EUGENE A. LOVELACE and J. DONALD SAVAGE, JR. \\ University of Virginia, Charlottesville, Virginia 22901
}

\begin{abstract}
Sixty-two Ss learned six CCC trigram-digit pairs by the study-test method; 21 Ss continued the PA acquisition trials to a learning criterion of one errorless trial, while $41 \mathrm{Ss}$ were carried to $300 \%$ overlearning or 24 trials, whichever came first. The Ss were then shown individual letters of the trigrams, with position cues provided, and were tested for their ability to provide the digit and produce the additional letters. Digit recall was much better to initial letters than to those in the second or third positions of the trigrams; with overlearning, digit recall improved to the initial letters but not to letters in second and third positions. Production of additional letters of the trigrams was not appreciably related to either cue position or degree of PA learning. There was some evidence of increased selectivity with higher degrees of PA learning.
\end{abstract}

It has clearly been established that Ss will selectively encode the elements of compound stimuli in a paired-associate (PA) learning task. An excellent review of this literature on stimulus selection has been provided by Richardson (1971). It has been reported that the selectivity of this encoding is reduced with higher degrees of training (e.g., James \& Greeno, 1967; Wichawut \& Martin, 1970). Wichawut and Martin (1970), for example, using word triads as compound stimuli, supported the notion that "When learning is carried beyond the list mastery level, it appears that more and more of the stimulus elements are additionally encoded as effective cues [p. 383]."

Lovelace and Blass (1968) found strong evidence of selective encoding with consonant trigrams as stimulus members of a PA task; as had been previously reported by Postman and Greenbloom (1967), the initial letter was the most effective cue. Lovelace and Blass also manipulated degree of original learning and reported that overlearning seemed to benefit only the association between the selected first letter and the digit response, "quite as if $S$ successfully ignored second and third letters once he began selection [p. 600]."

One purpose of the present study was to assess whether the failure of Lovelace and Blass to obtain an increase in effective use of additional cues of the trigrams with overlearning resulted from an insufficient manipulation of the variable, i.e., from too low a degree of overlearning. Their highest degree of training was 50\% overlearning; perhaps with a higher degree of overlearning, additional letters would become effective cues. To test this, two groups of Ss in the present study learned six trigram-digit pairs. One group of 21 Ss was run first to the criterion of one errorless trial to replicate the selection effects reported by Lovelace and Blass and to provide a baseline to which the performance of Ss given a high degree of overlearning might be compared.

\footnotetext{
* Requests for reprints should be addressed to E. A. Lovelace, Department of Psychology, Gilmer Hall. University of Virginia, Charlottesville, Virginia 22901.
}

A second, larger group of Ss $(\mathbb{N}=41)$ was carried to $300 \%$ overlearning or a total of 24 trials, whichever came first. A high degree of overlearning was desirable, but the practical constraints of the Ss' schedules prevented running all Ss to the $300 \%$ criterion; a large number of Ss were run in this condition so that the data could be separately analyzed for those who did and did not reach that high criterion, with a sizable number of Ss of both sorts (Ns of 15 and 26 resulted).

A second major purpose of this study was to determine the degree of functional independence of the elements of consonant trigram stimuli. Martin (1971) has shown that the reduction in selectivity of stimulus encoding with word triads as stimuli results from the formation of additional direct associations between individual stimulus elements and the response term, not from interelement associations within each stimulus compound. For the present data, the probability of additional letter recall was examined in relation to amount of original learning conditionalized upon the recall of the digit response.

The data again demonstrate a selection strategy favoring the initial letter of a consonant trigram as the effective stimulus for PA learning, and, even at high degrees of overlearning, there was no evidence that additional letters were becoming effective stimuli. The conditional probabilities of additional letter recall were much higher for pairs where the digit could be recalled than for those where it was not, suggesting that recall of additional letters was primarily mediated by recall of the digit rather than attributable to interelement associations. As degree of original learning increased, there was a systematic reduction in the probability of producing additional letters, given that the digit response was not recalled.

\section{METHOD}

The Ss learned six trigram-digit pairs. The stimuli were consonantal trigrams of $45 \%$ association value (Witmer. 1935): response terms were single-digit numbers. No stimulus element 


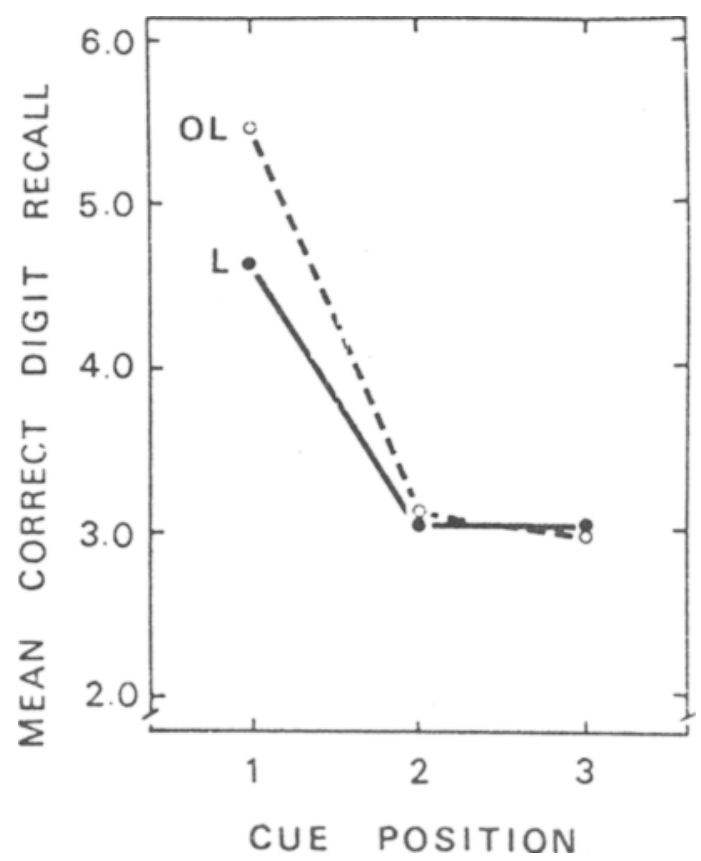

Fig. 1. Mean number of correct digit recalls across the three $T$ trials for Conditions $\mathrm{L}$ and $\mathrm{OL}$ as a function of the position of the letter cue presented.

(letter) was repeated within the list. The study-test method of PA learning was employed with three different orders of presentation; a 2-sec rate was used for both study and test trials, with a 2-sec blank interval between study and test and a 4-sec intertrial interval. Following PA learning, each $S$ was given three special test $(T)$ trials; on $T$ trials a $S$ attempted to recall the digit response and the missing letters when shown a single letter of a trigram. The missing stimulus elements were indicated by black squares, so the $\mathrm{S}$ had spatial information about the letter presented. On each of the three $T$ trials, each $S$ received the initial letters of two of the six pairs, the middle letters of another two pairs, and the terminal letter of the remaining two pairs. Across the three $T$ trials, each $S$ received each element of every stimulus; the order of the three $T$ trials was counterbalanced across Ss within each group. ${ }^{1}$ Stimuli were prepared as slides and presented by a Kodak Carousel 300 slide projector that was externally controlled by a Lafayette repeat-cycle timer.

For each of the $21 \mathrm{Ss}$ in Condition $\mathrm{L}$, the study-test procedure of PA learning was carried to a learning criterion of one errorless trial. For the $41 \mathrm{Ss}$ in Condition OL, the PA trials were continued to the criterion of $300 \%$ overlearning (i.e., four times the number of trials required to reach an errorless trial) or a maximum of 24 trials, whichever came first. After the test portion of the last PA trial, each S was read instructions for self-paced $T$ trials. Until this time, the $S$ had been given no indication that such special test trials involving individual stimulus elements would occur. The Ss were urged to guess at the digit and missing letters if they were uncertain. On a very few occasions, the $E$ found it necessary to ask the $S$ at the end of $45 \mathrm{sec}$ whether he wanted to give guesses as to the missing elements. Following the three $T$ trials, the $S$ was shown each of the six trigram-digit pairs and asked how he went about learning that pair.

The Ss were undergraduates at the University of Virginia; all but one of the $\mathrm{Ss}$ were enrolled in an introductory psychology course. None had prior laboratory experience with a PA task or with consonant trigrams. All $\mathrm{Ss}$ in Condition $\mathrm{L}$ served prior to any $\mathrm{Ss}$ being run in Condition OL, but all were drawn from the same classes and are presumed representative of the same population. This provided a determination of the distribution of trials to the criteria of one perfect performance and permitted a basis for determining a realistic overlearning criterion for use in running $S s$ in the OL condition. The data from a few Ss were discarded for failure to follow instructions, prior laboratory experience, or E error; no S's data were discarded for reasons of his performance.

\section{RESULTS AND DISCUSSION}

The mean numbers of trials to reach the criterion of one errorless trial were $6.76(\mathrm{SD}=5.06)$ and 7.22 $(S D=4.39)$ for Conditions $\mathrm{L}$ and $\mathrm{OL}$, respectively; the range of scores were identical for the two groups and the difference does not approach significance $[\mathrm{t}(60)=.35$, $\left.\sigma_{\overline{\mathrm{X}}}=1.30\right]$. The two groups of Ss can be presumed to be of equal learning ability.

In the $\mathrm{OL}$ condition, 26 of the 41 Ss reached the errorless criterion by Trial 6 and, thus, reached 300\% overlearning within 24 trials. For the remaining $15 \mathrm{Ss}$, the mean percent overlearning achieved when the PA task was terminated at the 24 th trial was $122 \%$, and only three Ss received less than $100 \%$ overlearning.

The frequencies of recall of additional letters and the numbers of correct digits recalled at each cue position did not change in any systematic way over the three $T$ trials. ${ }^{2}$ All summaries and analyses to be reported were performed on the combined data from the three $T$ trials.

The relative effectiveness of elements in the three positions as cues for recall of the digit responses during $\mathrm{T}$ trials is shown in Fig. 1. Recall was significantly related to cue position $[\mathrm{F}(2,120)=54.24$, MS error $=$ $1.77, p<.001]$. Neither degree of learning nor its interaction with position were statistically reliable sources of variance. The data support previous findings of selective encoding favoring the letters in the first (leftmost) position. This overall pattern of selectivity was also reflected in the relationship of digit recall to cue position for the majority of the individual Ss. In Condition $\mathrm{L}$ letters in the first position were most effective for 12 of the 21 Ss and tied for most effective for another 6 Ss. For Condition OL, first-position cues were most effective for 31 of the 41 Ss and tied for most effective for another $7 \mathrm{Ss}$. Verbal reports of the Ss as to how they had learned each pair further support this pattern of selective encoding. The Ss most frequently reported attempting to associate the first letter of the stimulus with the digit response; this was equally true for Conditions $\mathrm{L}$ and $\mathrm{OL}$.

Although overlearning does improve digit recall for cues in Position $1[\mathrm{t}(60)=3.78, \sigma \overrightarrow{\mathrm{X}}=.22, \mathrm{p}<.001]$, it does not enhance performance to cues in the second and third positions. There is, in other words, additional learning of responses to elements in the first position but no evidence that stimulus elements in the second or third positions become effective with overlearning. This is contrary to the findings of Wichawut and Martin (1970) and Martin (1971) when the stimulus elements 
were words: it is completely consistent with the findings of Lovelace and Blass (1968) where consonant trigrams were the stimulus materials.

The data for recall of additional stimulus elements indicates that $S$ s were neither integrating the stimulus nor associating the individual elements with the digit so that letter recall could be mediated via digit recall. The proportion of the other stimulus elements that were recalled to cues in Positions 1,2 , and 3 were .38, .44, and .43 , respectively, for Condition $\mathrm{L}$ and $.39, .41$, and .42 for Condition OL. Clearly, there was no increase in recall of additional stimulus elements with overlearning.

The number of occasions on which zero, one, or two additional letters were recalled to cues in Positions 1, 2, and 3 are shown for Conditions $L$ and OL in Table 1 . Also shown are the conditional probabilities of digit recall at each cue position given the number of additional letters correctly recalled. First, it can be noted with respect to recall of additional letters that the most probable outcome was for $S$ to be unable to recall any additional letters correctly. This finding is equally true for Conditions $\mathrm{L}$ and $\mathrm{OL}$ and is found to about the same degree at each of the three cue positions.

The conditional probabilities of digit recall are of interest, since correct digit recall on those occasions on which the $\mathrm{S}$ can recall no additional letters must indicate that the cue alone was an effective stimulus. This conditional probability of digit recall when no letters were recalled is fairly high given a cue in Position 1 (.65 and .86 for Conditions $\mathrm{L}$ and OL, respectively) but is very low for cues in Positions 2 and 3 (.16 and .10 for Condition L and .07 and .04 for Condition OL). This provides strong evidence that letters in the first position of a consonant trigram are likely to become the functional stimuli, but letters in the middle and terminal positions are not. There is no evidence that cues in the second and third positions become more effective with overlearning. In fact, these conditional probabilities, though higher for Cue Position 1, were lower at Cue Positions 2 and 3 for Condition OL than for Condition L.

Although not of central interest in the present study, a supplemental analysis of data for Ss in Condition OL provides some evidence concerning the relationship of selection strategy to learning ability. Are slow learners doing something different than fast learners? Are they doing poorly because they fail to employ any selective encoding strategy? The data for Condition OL in Table 1 were separated into that for Ss who reached criterion in six or fewer trials $(\mathrm{N}=26)$ and that for the slower learners who took more than six trials to reach the errorless criterion $(\mathrm{N}=15)^{3}$. Although it was obvious that the slow learners recalled fewer additional letters than the fast learners, the pattern of conditional probabilities of correct digit recall were highly similar for the fast and slow learners and similar to those in Table 1. It is apparent that the pattern of selective encoding of slow learners in this PA task is not
Table 1

Conditional Probabilities (p) of Digit Recall at Each Cue Position, Given the Mean Number (N) of Occurrences of 0,1 , or 2 Letters Correctly Reproduced

\begin{tabular}{|c|c|c|c|c|c|c|}
\hline \multirow{3}{*}{$\begin{array}{l}\text { Letters } \\
\text { Pro- } \\
\text { duced }\end{array}$} & \multicolumn{6}{|c|}{ Cue Position } \\
\hline & \multicolumn{2}{|c|}{1} & \multicolumn{2}{|c|}{2} & \multicolumn{2}{|c|}{3} \\
\hline & $\mathrm{N}$ & $\mathrm{p}$ & $\mathrm{N}$ & $\mathrm{p}$ & $\mathbf{N}$ & $\mathrm{p}$ \\
\hline \multicolumn{7}{|c|}{ Condition $\mathrm{L}(\mathrm{N}=21)$} \\
\hline 0 & 3.09 & .65 & 2.43 & .16 & 2.86 & .10 \\
\hline 1 & 1.24 & .81 & 1.86 & .62 & 1.14 & .83 \\
\hline 2 & 1.67 & .97 & 1.71 & .89 & 2.00 & .88 \\
\hline \multicolumn{7}{|c|}{ Condition OL $(\mathrm{N}=41)$} \\
\hline 0 & 3.07 & .86 & 2.93 & .07 & 2.98 & .04 \\
\hline 1 & 1.17 & .92 & 1.27 & .88 & 1.02 & .83 \\
\hline 2 & 1.76 & .99 & 1.80 & .99 & 2.00 & .99 \\
\hline
\end{tabular}

discernably different from that of fast learners (cf. Martin, 1971, p. 717); they both select the initial element.

None of the analyses presented above is directed to the question of independence of the functioning of the stimulus elements. There is selective encoding favoring the initial letter and evidence that additional elements neither become recallable themselves nor come to serve as effective cues for digit recall due to overlearning. The question remains, however, when Ss recalled some additional letters, whether these correct letters were elicited by the cue presented or were mediated by an independent, direct associative bond with the response term. Martin (1971) has clearly shown that such independent, direct associative bonds of elements to the response term, rather than interelement associations, is what occurs when the compound stimulus is a word triad. Figure 2 shows the probabilities of recall of one or both of the additional letters, conditional upon whether or not the response was correctly given.

The probabilities of recalling additional letters were much higher when the correct digit was recalled than when it was not, suggesting that much of the letter production might be mediated via direct associations to the digit responses. There are two aspects of this figure in which it differs notably from the corresponding plot (Fig. 1) of Martin's (1971) article. For one thing, there is no increase in recall of one or both of the other stimulus elements with increases in degree of learning, given that the $S$ successfully recalled the response term. This finding simply corroborates the other evidence that there is no increase in learning of additional stimulus elements with overlearning in the present study.

The second discrepancy between the present figure and Martin's corresponding plot is more puzzling. Martin found the probability of recall of other stimulus elements to be near zero at all levels of learning when the $S$ failed to recall the response term. Although this finding is approximated in the present study for the Ss in Condition OL, there was considerable recall of other letters by $S s$ in Condition $L$ when they failed to recall 


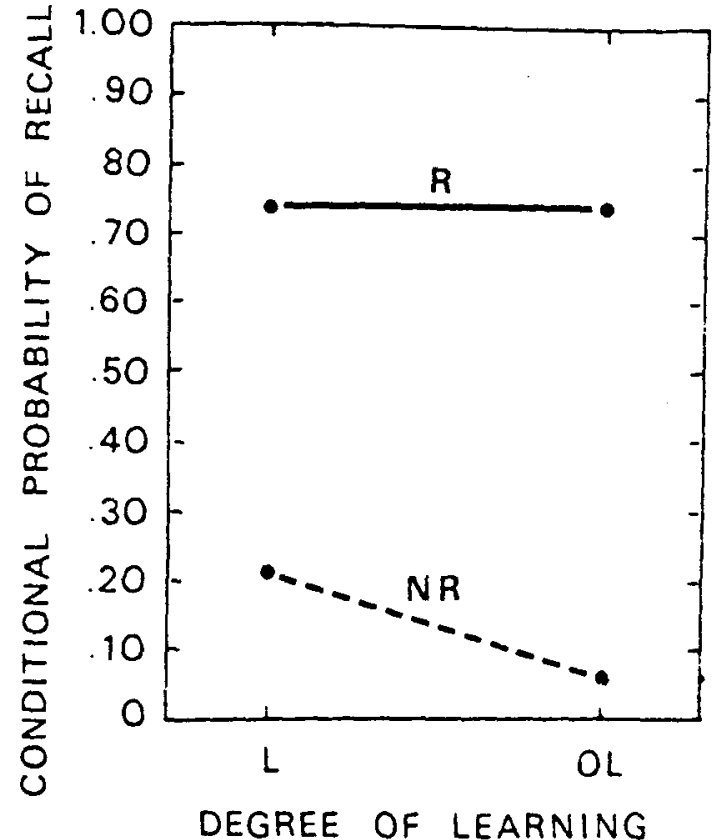

Fig. 2. Conditional probabilities of recalling at least one of the remaining two letters, given that the response digit was correctly recalled $(R)$ or was not recalled (NR) for Conditions $L$ and $O L$.

the response. A test for the equality of two percentages (Sokal \& Rohlf, 1969, p. 607) indicated that this drop in additional letter production, with increased degree of original learning when the digit could not be recalled $(22.1 \%$ vs $7.5 \%)$, was highly reliable $\left(t_{s}=4.19\right.$, $\mathrm{p}<.001)^{4}$

These data seem to suggest that interelement associations exist to some degree at the time the $S$ reaches the learning criterion of one errorless trial but that these associations are weakened appreciably during the course of overlearning. As a further check on whether this reduction in additional letters recallable in the absence of the correct response was systematically related to degree of original learning, the following analysis was performed. The number of correct responses during the PA learning was determined for each pair for each S. Ignoring the condition (L or OL) that the $S$ was in, these S-item combinations were sorted into four levels of original learning: those for which 1 to 5 correct responses were made during the PA learning, those with 6 to 13 correct responses, those with 14 to 18 correct responses, and those with 19 to 24 . The conditional probabilities of recall of additional letters when the correct response was not given were found to be $.205, .143, .090$, and .063 for the four levels of increasing degree of original learning, respectively. ${ }^{5}$ It appears that this drop in additional letter production is indeed systematic.

Two plausible interpretations can be offered. It may be that some stimulus items are moderately easy to integrate for some Ss, albeit for idiosyncratic reasons. If one assumes that such items are more meaningful to the $\mathrm{S}$, then PA learning of these pairs will occur relatively quickly. As one gets to higher degrees of original learning, it becomes increasingly unlikely that elements of these stimuli will fail to produce the correct digit. Thus, considering the data for occasions where the digit was not recalled, the proportion of stimuli for which some interelement associations exist for the $S$ are systematically reduced with increasing degree of original learning.

A second possible interpretation, and one of greater theoretical interest, is the possibility that the selectivity of encoding of elements of consonant trigram stimuli in a PA task actually increases with practice. The Ss may initially attend to a larger number of the elements of such stimulus compounds and then show an increased tendency to focus on single elements. In this connection, it should be recalled that the conditional probabilities of giving the correct digit response to cues in Positions 2 and 3 when no additional letters were recalled was greater for Condition $\mathrm{L}$ than for Condition OL. The combined data for responses to these two cue positions shows a drop from $12.6 \%$ in Condition $\mathrm{L}$ to $5.4 \%$ in Condition OL. This difference in percentages was tested in the manner proposed by Sokal and Rohlf (1969, p. 607) and found to be significant $\left(\mathrm{t}_{\mathrm{s}}=2.241\right.$, $\mathrm{p}<.025$ ). This is clearly consistent with the notion that there is a reduction in the breadth of functional cues with higher degrees of original learning.

The present data make it clear that any statement regarding the effects of stage of learning on selectivity of encoding stimulus elements must be conditional upon the nature of the stimulus compound. Although associations involving additional elements of word triad stimuli are learned during overlearning in a PA task, it is clear that this is not true for letter triads as stimuli. Indeed, once the Ss begin selective encoding of elements of consonant trigrams, this selectivity appears very stable and may even increase with overlearning.

\section{REFERENCES}

James, C. T., \& Greeno, J. G. Stimulus selection at different stages of paired-associate learning. Journal of Experimental Psychology, 1967, 74, 75-83.

Lovelace, E. A., \& Blass, E. M. Utilization of stimulus elements in paired-associate learning. Journal of Experimental Psychology, 1968, 76, 596-600.

Martin, E. Stimulus component independence. Journal of Verbal Learning \& Verbal Behavior, 1971, 10, 715-721.

Postman, L., \& Greenbloom, R. Conditions of cue selection in the acquisition of paired-associate lists. Journal of Experimental Psychology, 1967, 73, 91-100.

Richardson, J. Cue effectiveness and abstraction in paired-associate learning. Psychological Bulletin, 1971, 75, 73-91.

Sokal, R. R., \& Rohlf, F. J. Biometry. San Francisco: Freeman, 1969.

Wichawut, C., \& Martin, E. Selective stimulus encoding and overlearning in paired-associate learning. Journal of Experimental Psychology, 1970, 85, 383-388

Witmer, L. R. The association value of three-place consonant syllables. Journal of Genetic Psychology, 1935, 47, 337-360. 


\section{NOTES}

1. In Condition OL $(N=41)$, this was not a perfect counterbalancing, of course; here 14,14 , and 13 Ss received the three orders.

2. These two tables are available from the first author upon request.

3. These two tables are also available from the first author upon request.

4. The numbers of S-jtem combinations on which these percentages were based are 154 for Condition $L$ and 265 for Condition OL.

5. The numbers of S-item combinations on which these probabilities were based are $117,91,100$, and 111 for the four levels of increasing PA learning, respectively.

(Received for publication February 5, 1973; revision received April 4, 1973; accepted April 8, 1973.) 